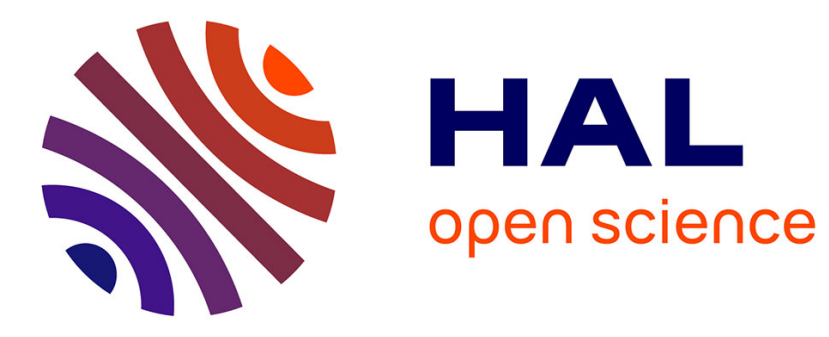

\title{
Light field denoising using 4D anisotropic diffusion
}

Pierre Allain, Laurent Guillo, Christine Guillemot

\section{To cite this version:}

Pierre Allain, Laurent Guillo, Christine Guillemot. Light field denoising using 4D anisotropic diffusion. ICASSP 2019 - IEEE International Conference on Acoustics, Speech, and Signal Processing, May 2019, Brighton, United Kingdom. pp.1692-1696, 10.1109/ICASSP.2019.8682709 hal-02116372

\section{HAL Id: hal-02116372 https://hal.science/hal-02116372}

Submitted on 30 Apr 2019

HAL is a multi-disciplinary open access archive for the deposit and dissemination of scientific research documents, whether they are published or not. The documents may come from teaching and research institutions in France or abroad, or from public or private research centers.
L'archive ouverte pluridisciplinaire HAL, est destinée au dépôt et à la diffusion de documents scientifiques de niveau recherche, publiés ou non, émanant des établissements d'enseignement et de recherche français ou étrangers, des laboratoires publics ou privés. 


\title{
LIGHT FIELD DENOISING USING 4D ANISOTROPIC DIFFUSION
}

\author{
Pierre Allain, Laurent Guillo, Christine Guillemot \\ Inria Rennes Bretagne-Atlantique, 263 Avenue Général Leclerc, 35042 Rennes
}

\begin{abstract}
In this paper, we present a novel light field denoising algorithm using a vector-valued regularization operating in the 4D ray space. More precisely, the method performs a PDE-based anisotropic diffusion along directions defined by local structures in the $4 \mathrm{D}$ ray space. It does not require prior estimation of disparity maps. The local structures in the 4D light field are extracted using a 4D tensor structure. The paper then describes the strategy retained for setting the diffusion tensor parameters for the targeted denoising application. It then analyzes the influence of the model parameters on the denoising performance. Experimental results show that the proposed denoising algorithm performs well compared to state of the art methods while keeping tractable complexity.
\end{abstract}

Index Terms-Light Field, regularization, anisotropic diffusion, denoising

\section{INTRODUCTION}

Light field imaging has emerged as a promising technology for a variety of applications including photorealistic rendering, computational photography and computer vision. Various camera designs have been proposed to capture real light fields going from camera arrays [1], to single cameras mounted on moving gantries, and plenoptic cameras [2], [3]. These camera architectures present different characteristics in terms of spatial and angular resolution. Plenoptic cameras rely on multiplexing and use an array of microlenses placed in front of the sensor to capture multiple low resolution views in one 2D sensor image. This easy way to capture multiple view-points, suffers from a low spatial resolution and reduced light throughput, resulting in visible noise artefacts, compared to $2 \mathrm{D}$ classical cameras.

This paper addresses the problem of denoising in dense light fields. Denoising is an ill-posed problem which, to be solved, requires introducing some prior knowledge on the kind of typical images we try to restore, which helps restricting the class of admissible solutions. The authors in [4] use a Gaussian mixture model (GMM) prior for light field patches, and then exploit this prior within a Bayesian inference framework for light field denoising. In [5], the light field is denoised by stacking EPIs in a 3D volume using the VBM4D video denoising method [6], while the authors in [7] apply a hyper-fan shaped filter in a 4D discrete Fourier transform domain. The BM3D denoising algorithm [8] is extended in [9] to light fields.

. This work was supported by the EU H2020 Research and Innovation Programme under grant agreement No 694122 (ERC advanced grant CLIM)
Smoothness priors have been widely used together with regularization schemes for inverse problems in 2D imaging such as denoising. The only approach of regularization proposed so far for light field denoising is the one of [10] using a global variational framework integrating contributions of regularizers on vector fields computed in EPI 2D spaces. The authors use 2D structure tensors computed in epipolar plane images (EPI) to estimate disparity between light field views. With the help of the estimated disparity, they project all the views on a target viewpoint and solve the regularization using a global optimization framework with total variation priors. The final regularizer is written as the contributions of regularizers on vector fields in EPI 2D spaces, with user-defined constants which adjust the amount of smoothing on the different views or EPIs. Errors in disparity maps may however translate into annoying artefacts in particular along edges.

In this paper, we propose instead a regularization method operating directly in the 4D ray space that does not require prior estimation of disparity maps. The method performs a PDE-based diffusion with anisotropy steered by a tensor field based on local structures in the $4 \mathrm{D}$ ray space that we extract using a light field 4D tensor structure. To enhance coherent structures, the smoothing along directions, surfaces, or volumes in the 4D ray space is done with a diffusivity coefficient that is derived from the amount of local variations in the 4D space. More precisely, the diffusivity coefficient is computed as a function of the 4 eigenvalues of the 4D structure tensor. The diffusion is isotropic in homogenous regions with no strong structures, whereas it follows structures in non homogenous regions.

The proposed regularization model has been applied for denoising light fields from the EPFL dataset [11]. Experimental results show that the proposed method compares well to the best state of the art method [9], even outperforming it at high noise levels, while being considerably faster. We refer to our method as Anisotropic Diffusion Denoising 4D (ADD4D).

\section{4D ANISOTROPIC DIFFUSION}

Let $L(x, y, u, v)$ denote the $4 \mathrm{D}$ representation of a light field, describing the radiance of a light ray parameterized by its intersection with two parallel planes [12], and where $(u, v)$ denote the angular (view) coordinates and $(x, y)$ the spatial (pixel) coordinates.

\subsection{D structure tensor}

To perform light field regularization along pre-defined directions which would preserve edges, one has to first characterize local 


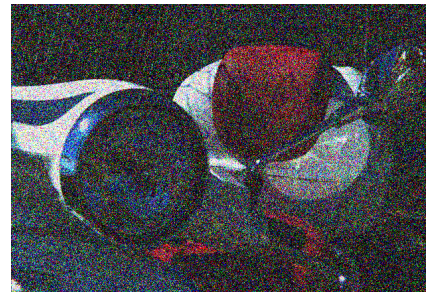

(a)

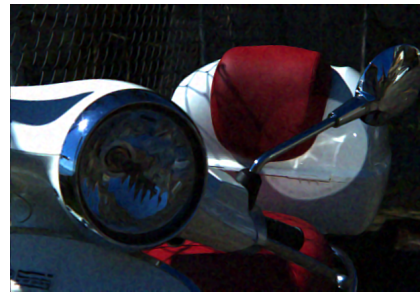

(b)

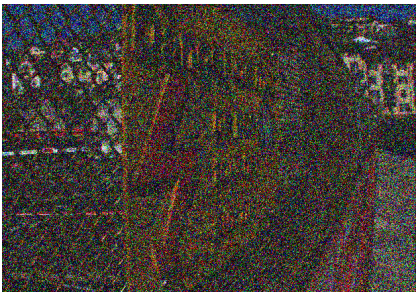

(c)

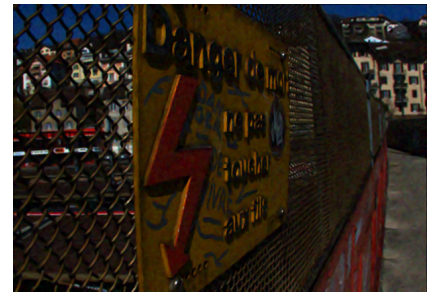

(d)

Fig. 1: 4D tensor driven diffusion is able to denoise highly damaged light fields. Center views for Vespa and DangerDeMort from the EPFL dataset. (a,c) With Additive Gaussian noise ( $\sigma=\frac{100}{255}$, PSNR= $8.13 \mathrm{~dB}$ ); (b,d) After denoising giving global PSNR on all subapertures of $29.31 \mathrm{~dB}$ (b) and $28.50 \mathrm{~dB}$ (d).

structures in the image, or here the light field. While light field regularization methods are usually applied per Epipolar Plane Image (EPI) of the light field with directions given by 2D structure tensors often smoothed with a 2D Gaussian kernel, we consider instead a direct computation of 4D structure tensors in the $4 \mathrm{D}$ ray space, which is defined as the symmetric and semipositive definite $4 \times 4$ matrix [13]:

$$
\mathbf{T}=\frac{1}{C} \sum_{c=1}^{C} \nabla L_{c} \otimes \nabla L_{c}^{\top},
$$

where $C$ is the number of color channels. The notation $\nabla L_{c}$ denotes the gradient or partial derivatives in the 4 dimensions of the color channel $L_{c}$. A smoothed version $\mathbf{T}_{\omega}=$ $\mathbf{T} * G_{\omega}(x, y, u, v)$ of the structure tensor is in practice computed to retrieve a more coherent geometry, where $G_{\omega}(x, y, u, v)$ is a Gaussian kernel smoothing along the four light field dimensions. Its spectral elements, i.e. the eigenvectors $\nu_{i}, i=1 \ldots 4$ and the eigenvalues $\lambda_{i}, i=1 \ldots 4$ of the $4 \mathrm{D}$ structure tensor $\mathbf{T}$ respectively give the orientations of the local structures in the $4 \mathrm{D}$ rays space and the vector-valued variations along these structures. We therefore have:

$$
\mathbf{T}_{\omega}=\sum_{i=1}^{4} \lambda_{i} \nu_{i} \nu_{i}^{T}
$$

The eigenvector $\nu_{1}$ with the largest eigenvalue $\lambda_{1}$ gives the dominant orientation of the local structures in the 4D ray space. The ratios between the eigenvalues gives information on the type of structure present in the local neighborhood.

\section{TRACE-BASED PDE DIFFUSION}

We consider a trace-based PDE as introduced in [14] for 2D images, which we here extend to 4D light fields as:

$$
\frac{\partial L_{c}}{\partial t}=\operatorname{trace}\left(\mathbf{D H}_{c}\right)
$$

where $\mathbf{H}_{\mathbf{c}}$ is the $4 \mathrm{D}$ Hessian matrix of the $c$ channel of $L$, defined as:

$$
\mathbf{H}_{\mathbf{c}}=\left(\begin{array}{cccc}
\frac{\partial^{2} L_{c}}{\partial x^{2}} & \frac{\partial^{2} L_{c}}{\partial x \partial y} & \frac{\partial^{2} L_{c}}{\partial x \partial u} & \frac{\partial^{2} L_{c}}{\partial x \partial v} \\
\frac{\partial^{2} L_{c}}{\partial y \partial x} & \frac{\partial^{2} L_{c}}{\partial y^{2}} & \frac{\partial^{2} L_{c}}{\partial y \partial u} & \frac{\partial^{2} L_{c}}{\partial y \partial v} \\
\frac{\partial^{2} L_{c}}{\partial u \partial x} & \frac{\partial^{2} L_{c}}{\partial u \partial y} & \frac{\partial^{2} L_{c}}{\partial u^{2}} & \frac{\partial^{2} L_{c}}{\partial u \partial v} \\
\frac{\partial^{2} L_{c}}{\partial v \partial x} & \frac{\partial^{2} L_{c}}{\partial v \partial y} & \frac{\partial^{2} L_{c}}{\partial v \partial u} & \frac{\partial^{2} L_{c}}{\partial v^{2}}
\end{array}\right)
$$

and $\mathbf{D}$ is a diffusion tensor to be defined according to the regularization goals.
To be adapted to the local structures in the 4D ray space of the light field, the diffusion process must be driven by a specific tensor, called diffusion tensor chosen so that it has the same set of eigenvectors $\nu_{i}, i=1 \ldots 4$ as the structure tensor $\mathbf{T}$. A diffusion tensor is therefore constructed as:

$$
\mathbf{D}=\sum_{i=1}^{4} \alpha_{i} \nu_{i} \nu_{i}^{T},
$$

where the coefficient $\alpha_{i}$ set the smoothing strength along the direction given by $\nu_{i}$, and are chosen according to the goal of the diffusion.

\subsection{Setting the diffusion tensor parameters}

For denoising purpose, the coefficients $\alpha_{i}$ controlling the strength of the diffusion have to depend on the quantity of local variation. Considering $\psi=\sqrt{\sum_{i=1}^{4} \lambda_{i}}$ as a measure of local variation [15], we set these parameters as $\alpha_{i}=f_{i}(\psi)$. The functions $f_{i}(\psi)$ are chosen to abide by denoising goal. Firstly, the diffusion should be isotropic in regions where there is no apparent direction, i.e. where $L$ is homogeneous:

$$
\lim _{\psi \rightarrow 0} \mathbf{D}=a \mathbf{I} \Rightarrow f_{1}(0)=f_{2}(0)=f_{3}(0)=f_{4}(0) .
$$

Conversely, in regions where structures are clearly appearing, no diffusion must be performed in order to preserve geometry, that is:

$$
\lim _{\psi \rightarrow \text { inf }} \mathbf{D}=0 \Rightarrow f_{1}(\psi)=f_{2}(\psi)=f_{3}(\psi)=f_{4}(\psi)=0 .
$$

Also, the diffusion must be performed along the orientations of the local structures, given by the eigenvectors having the lowest eigenvalues. We assume that more structure there is, more the eigenvectors corresponding to the lowest eigenvalues must drive the diffusion. For instance, for a high quantity of structure, the diffusion should only be performed in the $\nu_{4}$ direction. In other words, if the structure is clearly shaped, the diffusion should only correct structure singularities with the highest degree. Applying such reasoning to the other eigenvectors, we obtain the condition:

$$
f_{1}(\psi) \leq f_{2}(\psi) \leq f_{3}(\psi) \leq f_{4}(\psi) .
$$

We therefore propose the function $f_{i}$ meeting the conditions of equations 6,7 and 8:

$$
f_{i}(\psi)=e^{-\left(\frac{\psi}{\rho_{i}}\right)^{\gamma}}, \text { with } \rho_{1} \leq \rho_{2} \leq \rho_{3} \leq \rho_{4}
$$


where $\rho_{i}$ is a parameter related to the quantity $\psi$ of local variations corresponding to a point where the diffusion strength $f(\psi)$ significantly varies. The parameter $\gamma$ defines the exponential behaviour of the function. In the particular case where $\gamma=2$, the parameter $\rho_{i}$ corresponds to the standard deviation of the function $f(\psi)$. This parameterization allows the tuning of the diffusion process on the basis of structure properties.

\subsection{Model tuning}

We discuss here the influence of the model parameters for denoising. Instead of parameterizing independently every $\rho_{i}$, we propose the following expression:

$$
\rho_{i}=\rho_{0} \alpha^{i-1}
$$

which allows a simplification of the model to two parameters. Figure 3 plots different functions $f_{i}(\psi)$ parameterized by $\rho_{i}$. We set $\alpha$ and $\gamma$ to 2 and study the influence of $\rho_{0}$ on the denoising process. One can note that $\alpha$ is related to an anisotropic ratio, while $\rho_{0}$ is related to a local variation intensity.

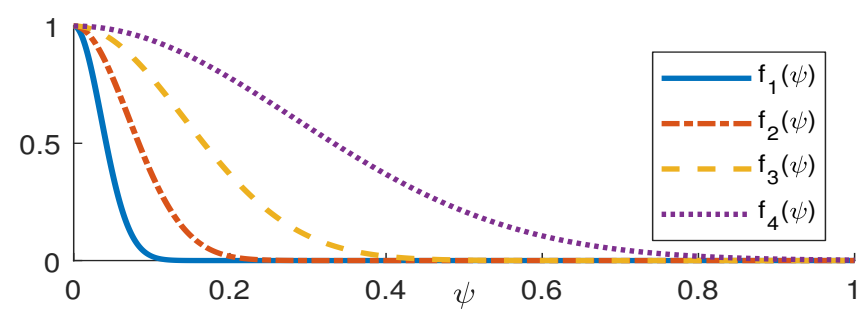

Fig. 3: Functions $f_{i}(\psi)$ controlling smoothing strength of the diffusion tensor along orientations defined by the four eigenvectors $\nu_{i}$, with $\rho_{0}=$ 0.05 .

We present denoising results with different set-ups for a single light field. The model integration is performed using the same time step of 0.1 to raise parameters influence of the denoising. Fig. $2 \mathrm{a}$ and $2 \mathrm{~b}$, show that, for low and medium noise $\left(\sigma=\frac{10}{255}, \sigma=\frac{40}{255}\right.$ ), a low value of $\rho_{0}$ (respectively 0.01 and $0.03)$ prevents the model to damage the light field structures. On the other hand, a low value of $\rho_{0}$ is not suitable for high noise levels as shown in $2 \mathrm{~d}$, where the model is unable to diffuse along the structures, hence many iterations are needed to remove the noise. For high values of $\rho_{0}$, we can observe a more efficient behaviour regarding noise reduction. But in this case, the model can damage the structures (more than with low values of $\rho_{0}$ ), as we can see when the number of iterations increases.

Fig. 2d-f show that, for the different values of the parameter $\rho_{0}$, when increasing the number of iterations, the model converges to the same PSNR curve, at both low and high noise level. This shows that, even for very different intensities of added noise, the model is mostly responsible for the new structures of the light field. In other words, noise is simply cleaned more or less quickly while the model is on its way to its intrinsic regularization evolution.

\begin{tabular}{|c||c|c|c|c|c|}
\hline Method & $\sigma=\frac{10}{255}$ & $\sigma=\frac{20}{255}$ & $\sigma=\frac{30}{255}$ & $\sigma=\frac{40}{255}$ & $\sigma=\frac{50}{255}$ \\
\hline HF4D [7] & 31.07 & 25.80 & 22.61 & 20.34 & 18.59 \\
\hline BM3D [8] & 35.42 & 32.85 & 31.36 & 30.25 & 29.32 \\
\hline BM3D Epi [17] & 36.09 & 33.48 & 31.90 & 30.71 & 29.67 \\
\hline VBM4D [18] & 36.07 & 33.52 & 31.92 & 30.67 & 29.63 \\
\hline VBM4D Epi [5] & 36.13 & 33.51 & 31.92 & 30.72 & 29.72 \\
\hline LFMB5D [9] & $\mathbf{3 6 . 5 0}$ & $\mathbf{3 4 . 2 1}$ & $\mathbf{3 2 . 8 7}$ & $\mathbf{3 1 . 8 4}$ & 30.99 \\
\hline \hline ADD4D & 36.13 & 33.88 & 32.67 & $\mathbf{3 1 . 8 4}$ & $\mathbf{3 1 . 1 9}$ \\
\hline
\end{tabular}

Table 1: Comparison of light field denoising methods (state of the art results are courtesy of [9]). PSNR in dB.

\section{DENOISING RESULTS}

The proposed denoising model is applied on 12 light fields of the EPFL dataset [11] extracted using Dansereau's Matlab light field toolbox [16] in the same conditions as in [9]. They are chosen to allow comparison to state of the art methods. Because of the strong irregularities in the angular dimension resulting from the vignetting effect, we chose to denoise only the subapertures that are not affected by the later and therefore limit the size of light fields to $11 \times 11$. Indeed, because of its regularization properties, our method would also correct artefacts at angular corners (i.e. the vignetting effect), hence the measured PSNR would not only reflect the denoising performance.

For our experiments we chose to use the same model parameterized by $\rho_{0}=0.05, \alpha=\gamma=2$, and tensor smoothing is set to $\omega=5$ in the spatial and angular directions. Integration of Eq. 3 is performed using the Runge-Kutta 4 scheme, and the time step is set to 0.1 . One iteration takes 7 minutes to compute on a i7-6600U without any parallelization, and best PSNR is usually obtained from 4 iterations to 20 depending on the light field and the model. For comparison, LFBM5D performs approximately in 7 hours on an octo core processor (but without angular cropping). On a 10 iterations basis for our method, this gives an estimated 26 times faster result. Detailed results are provided ${ }^{1}$ to show angular consistency. In figure 4 , we observe that our model is able to remove low noise while keeping fine original structures. Our model is also able to deal with high level of noise as shown in figures 1 and 5, where we can see in the last that the result qualitatively matches the best existing method. In table 1 we can see the denoising model we used outperforms other methods for a high level of noise, while comparing well for low levels.

\section{CONCLUSION}

In this paper we proposed a new approach for denoising light fields. Using structural properties in the four dimension space, we showed that tensor oriented diffusion can drastically reduce noise while keeping most of the structures of the subaperture images. We believe that this work can be extended with different denoising models depending on scene's disparities and structures, and can highly benefit of parallelization.

1. http://clim.inria.fr/research/ADD4D 


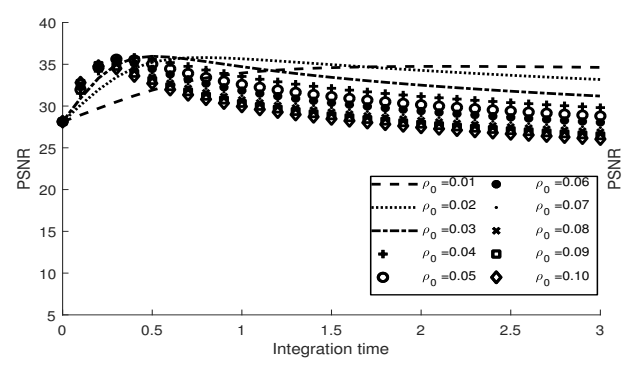

(a) Initial gaussian noise : $\sigma=\frac{10}{255}$

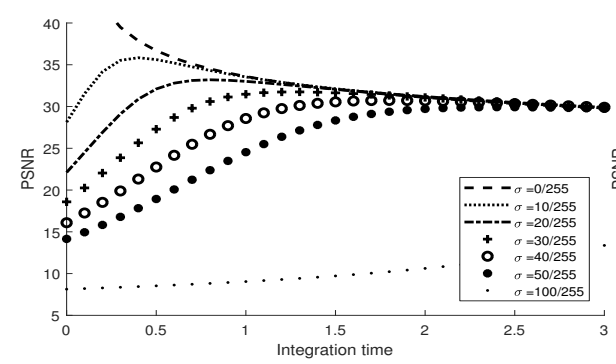

(d) Model parameter : $\rho_{0}=0.04$

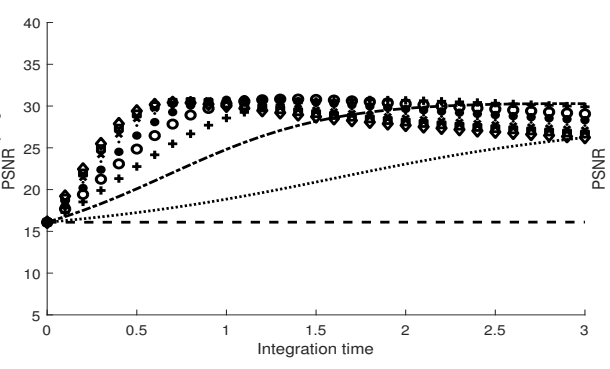

(b) $\sigma=\frac{40}{255}$

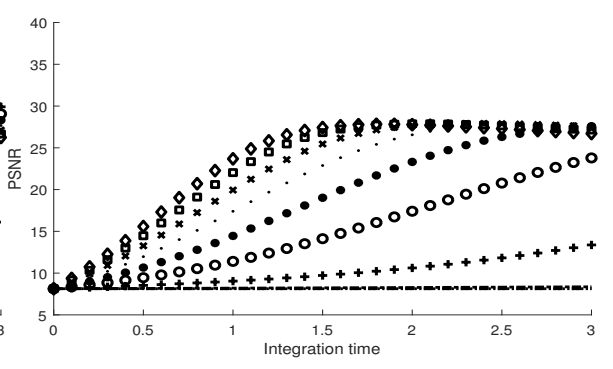

(c) $\sigma=\frac{100}{255}$

Fig. 2: Denoising behaviour for different noise levels applied to the Bike light field. Each curve represents the PSNR evolution along the iterations of the diffusion for: (top row) - different values of the parameter $\rho_{0}$ of the model, and for different noise levels For low and medium noise (a,b), we can see that the best PSNR is obtained for a low value of the parameter $\sigma_{0}$; (bottom row) - different noise levels, and for different values of the parameter $\rho_{0}$ of the model

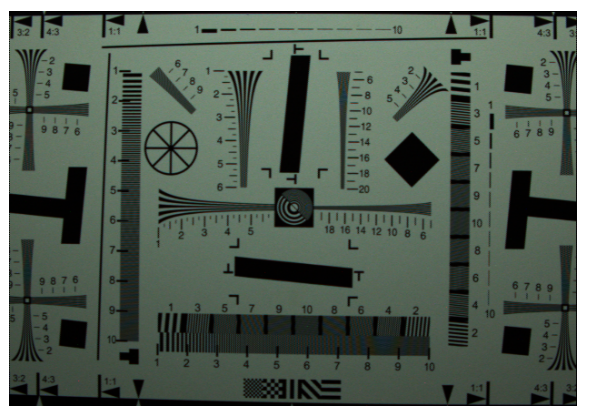

(a)

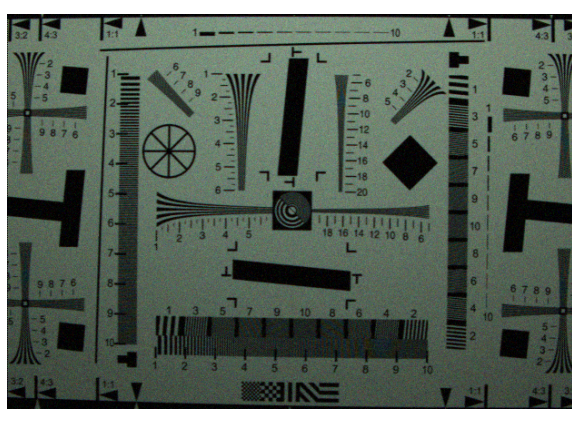

(b)

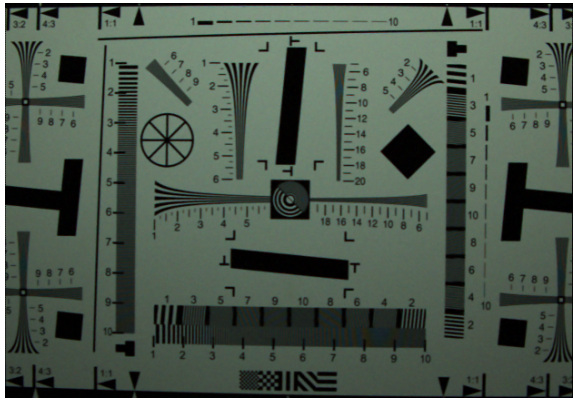

(c)

Fig. 4: Denoising of IsoChart light field. Center views. (a) Original. (b) Gaussian noise of $\sigma=\frac{10}{255}$, PSNR 28.13 dB. (c) Using our method, 4 iterations, PSNR $35.10 \mathrm{~dB}$.

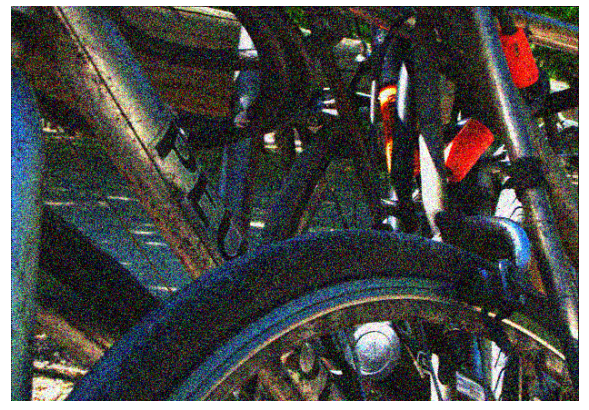

(a)

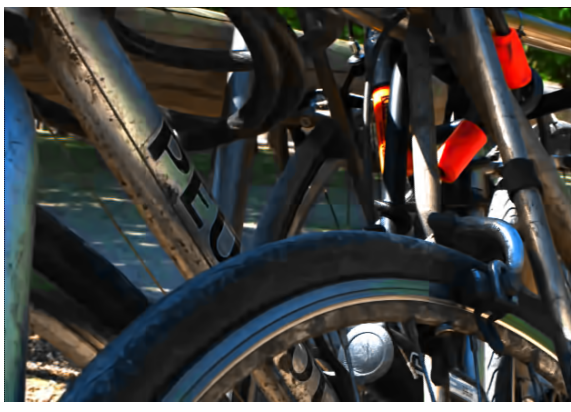

(b)

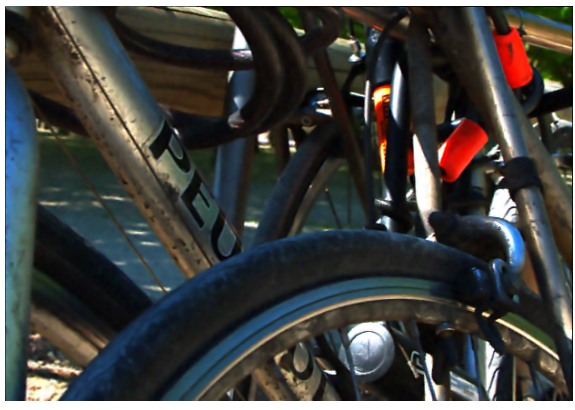

(c)

Fig. 5: Denoising of Bikes light field. Center views. (a) Gaussian noise of $\sigma=\frac{50}{255}$, PSNR $14.15 \mathrm{~dB}$. (b) Result obtained using LFBM5D, PSNR 29.91 dB. (c) Using our method, 19 iterations, PSNR $30.10 \mathrm{~dB}$. 


\section{REFERENCES}

[1] B. Wilburn, N. Joshi, V. Vaish, E.-V. Talvala, E. Antunez, A. Barth, A. Adams, M. Horowitz, and M. Levoy, "High performance imaging using large camera arrays," $A C M$ Trans. Graph., vol. 24, no. 3, pp. 765-776, July 2005.

[2] R. Ng, Light Field Photography, Ph.D. thesis, Stanford University, 2006.

[3] Todor Georgiev, Georgi Chunev, and Andrew Lumsdaine, "Superresolution with the focused plenoptic camera," 2011.

[4] K. Mitra and A. Veeraraghavan, "Light field denoising, light field superresolution and stereo camera based refocussing using a gmm light field patch prior," in Proc. CVPR Workshops, June 2012, pp. 22-28.

[5] A. epas Moghaddam, P. L. Correia, and F. Pereia, "Light field denoising: exploiting the redundancy of an epipolar sequence representation," in Proc. 3DTV-Con, Jul. 2016.

[6] G. Boracchi, A. Foi, and K. Egiazarian, "Video denoising, deblocking, and enhancement through separable 4-d nonlocal spatiotem- poral transforms," IEEE Trans. on Image Processing, vol. 21, no. 9, pp. 3952-3966, 2012.

[7] D. G. Dansereau, D. L. Bongiorno, O. Pizarro, and S.B. Williams, "Light field image denoising using a linear $4 \mathrm{~d}$ frequency-hyperfan all- in-focus filter," in Proc. SPIE, Feb. 2013, vol. 8657.

[8] K. Dabov, A. Foi, and K. Egiazarian, "Image denoising by sparse 3-d transform-domain collaborative filtering," IEEE Trans. on Image Processing, vol. 16, no. 8, pp. 2080-2095, 2007.

[9] M. Alain and A. Smolic, "Light field denoising by sparse $5 \mathrm{~d}$ transform domain collaborative filtering," in IEEE International Workshop on Multimedia Signal Processing, Oct. 2017.

[10] B. Goldluecke and S. Wanner, "The variational structure of disparity and regularization of $4 \mathrm{~d}$ light fields," in IEEE Conf. on Computer Vision and Pattern Recognition (CVPR), June 2013, pp. 1003-1010.

[11] Martin Rerabek and Touradj Ebrahimi, "New light field image dataset," in Proc. QoMEX, 2016.

[12] S.J. Gortler, R. Grzeszczuk, R. Szeliski, and M.F. Cohen, "The lumigraph.," 23rd annual conference on Computer graphics and interactive techniques, ACM, pp. 43-54, 1996.

[13] Joachim Weickert, "Coherence-enhancing diffusion of colour images," Image and Vision Computing, vol. 17, no. 3, pp. $201-212,1999$.

[14] P. Perona and J. Malik, "Scale space and edge detection using anisotropic diffusion," IEEE Trans. on Pattern Analysis and Machine Intelligence, vol. 12, pp. 629-639, 1990.

[15] David Tschumperlé and Rachid Deriche, "Anisotropic Diffusion Partial Differential Equations in Multi-Channel Image Processing : Framework and Applications.," in Advances in Imaging and Electron Physics (AIEP), pp. 145-209. Academic Press, 2007.
[16] D. G. Dansereau, O. Pizarro, and S. B. Williams, "Decoding, calibration and rectification for lenselet-based plenoptic cameras," in 2013 IEEE Conference on Computer Vision and Pattern Recognition, June 2013, pp. 10271034.

[17] Zeyu Li, H. Baker, and R. Bajcsy, "Joint image denoising using light-field data," in 2013 IEEE International Conference on Multimedia and Expo Workshops (ICMEW), July 2013, pp. 1-6.

[18] M. Maggioni, G. Boracchi, A. Foi, and K. Egiazarian, "Video denoising, deblocking, and enhancement through separable 4-d nonlocal spatiotemporal transforms," IEEE Transactions on Image Processing, vol. 21, no. 9, pp. 3952-3966, Sept 2012. 